\title{
Promote Assistive and AAC technologies during Covid-19
}

\section{Promuovere le tecnologie assistive e la CAA al tempo del Covid-19}

\author{
Michela Galdieria, Michele Domenico Todino ${ }^{\mathrm{b}, 1}$ \\ a Università degli Studi del Sannio di Benevento, michela.galdieri@unisannio.it \\ b Università degli Studi del Sannio di Benevento, micheledomenico.todino@unisannio.it
}

\begin{abstract}
The Covid-19 health emergency has produced a rethinking of education and training systems based on open and flexible physical spaces and remote communication channels; however, socialization processes and virtual relational exchanges are still possible and at the same time authentic. Moreover, the use of telecommunication technologies augment efforts to find a new way to organize educational spaces when it is not possible to share physical space and virtual spaces must be used. Starting from the role of assistive technologies in European policies, this work presents a case study about the inclusive perspective of corporeality and action in teaching-learning process and described an experience done in a third grade class of a primary school in Rome where a teacher used an eye communicator with GRID3 software and tools of Augmentative Alternative Communication with a student with complex communication needs, main goal of this activity was to create an inclusive and sharing path for each scholar done in distance education.
\end{abstract}

Keywords: assistive technologies; distance learning, inclusion, augmentative alternative communication.

Sintesi

L'emergenza sanitaria da Covid-19 ha sollecitato un ripensamento dei sistemi educativi e formativi quali dimensioni aperte e flessibili in cui formarsi, spazi nei quali i canali di comunicazione a distanza hanno reso possibile processi di socializzazione e scambi relazionali virtuali ma non per questo meno autentici, luoghi della didattica in cui favorire gli apprendimenti mediante l'uso di tecnologie che hanno consentito di raggiungere risultati anche in assenza di condivisione di uno spazio fisico. Il lavoro presenta una riflessione sul ruolo delle tecnologie assistive nelle politiche europee, sul potenziale inclusivo della corporeità e dell'azione nei percorsi di insegnamento-apprendimento e propone la descrizione di un'esperienza svoltasi nella classe terza di una scuola primaria romana dove, in presenza di un'alunna con gravi difficoltà comunicative, la didattica ha previsto l'uso del comunicatore oculare con software GRID3 coniugato alle pratiche e agli strumenti propri della Comunicazione Aumentativa Alternativa, con l'obiettivo di creare un percorso inclusivo e partecipativo per ciascun alunno, seppure a distanza.

Keywords: tecnologie assistive; didattica a distanza, inclusione, comunicazione aumentativa alternativa.

\footnotetext{
${ }^{1}$ The present study was conducted in full collaboration by the authors. However, M. Galdieri wrote $\S 3, \S 4, \S 5, \S 5.1$ and $\S 6$; M. D. Todino wrote $\S 1$ and $\S 2$.
} 


\section{Introduzione}

In Italia, dalla metà del secolo scorso, le istituzioni scolastiche e la ricerca pedagogica hanno prestato una crescente cura nel calibrare i percorsi di insegnamento-apprendimento sui reali bisogni educativi e formativi di ciascun alunno. Com'è noto, l'evolversi di questo processo ha posto le basi per superare l'idea di un'istruzione speciale e differenziale creando le occasioni affinchè la scuola potesse progressivamente approdare ad un paradigma inclusivo in un clima complessivo di apertura e innovazione in cui il concetto stesso di inclusione ha identificato sempre più spesso "un processo, una filosofia dell'accettazione, ossia la capacità di fornire una cornice dentro cui gli alunni - a prescindere da abilità, genere, linguaggio, origine etnica o culturale - possono essere ugualmente valorizzati, trattati con rispetto e forniti di uguali opportunità a scuola" (Dovigo, 2008, p. 13). Parte di questo cambiamento dovrebbe essere attribuito alle tecnologie didattiche (Calvani, 2007), digitali o analogiche, che hanno da sempre cercato di offrire strumenti vicarianti (Rivoltella, 2017; Sibilio, 2017a; 2017b) capaci di mettere in gioco nuove possibilità di accesso al sapere, nuove modalità di relazioni e vie alternative per favorire uno o più stili d'apprendimento. Le tecnologie, che incidono sul nostro modo di vivere e di partecipare alla vita sociale, possono prospettare nuovi orizzonti conoscitivi, di condivisione e di crescita culturale ed emotiva per tutti e rappresentare delle opportunità inclusive nel variegato panorama delle nostre scuole (MIUR, 2012). La Direttiva Ministeriale del 27 dicembre del 2012, e successive integrazioni, non a caso, ha identificato un'area dello svantaggio scolastico non riconoducibile alla sola disabilità; a locuzione bisogni educativi speciali, difatti, evidenzia le diverse difficoltà che possono manifestarsi nei percorsi relazionali e di apprendimento (Ianes, 2015) e, lungi dall'etichettare e dal medicalizzare, invita la scuola a saper riconoscere e a saper accogliere tempestivamente i segnali di un disagio con lo scopo di incentrare l'azione didattica su un insieme di buone pratiche, mediate anche dall'uso delle tecnologie, che non siano pensate per il singolo studente ma, in maniera trasversale, siano indirizzate all'intero gruppo classe (Cottini, 2017; Ianes \& Macchia, 2008), favoriscano processi di insegnamento-apprendimento e un'educazione inclusiva in contesti formali e non formali. In realtà, secondo Laurillard (2014) l'incredibile impatto che esse hanno avuto in ogni contesto, dal lavoro al tempo libero agli ambienti di apprendimento "ha costituito uno shock ancora da assorbire [...]la varietà e la potenzialità delle tecnologie digitali fanno sì che esse non possano essere inglobate facilmente - sarà piuttosto il sistema nel suo complesso che dovrà modificarsi per abbracciarle completamente" (p. 16); nello specifico, considerato l'effetto che esse hanno e avranno sugli itinerari educativi e formativi, è necessario un cambio di prospettiva da parte dei docenti che dovrebbero conoscerle e saperle utilizzare, per poterne sfruttare a pieno le potenzialità e metterle al servizio dell'educazione. L'emergenza epidemiologica, purtroppo ancora in corso, ha rappresentato un momento di rottura forte nel tradizionale modo di intendere la didattica ma, nel contempo, un'occasione per le istituzioni scolastiche per ripensarsi su nuove basi e tentare di governare l'inatteso (Weick, Kathleen \& Sutcliffe, 2010) ponendosi come realtà organizzative in cui maturare una piena consapevolezza delle potenzialità delle proprie risorse, dei propri mezzi e dei propri strumenti, ivi comprese le tecnologie che hanno consentito di raggiungere risultati "insperati" anche in assenza di condivisione di uno spazio fisico (Lazzari, 2017, p. 122); un'emergenza che ha avviato una profonda riflessione sul tema della "flessibilità e dell'adattamento al cambiamento" (Berthoz, 2011, p. 9). Il rapporto tra tecnologia ed educazione a distanza (Bertagna, 2020, Calvani, 2007) richiede, infatti, azioni didattiche che non trascurino i processi cognitivi, il vissuto emotivo, le dinamiche relazionali e l'opportunità di ciascun studente di fare esperienza attraverso il proprio corpo. Non si tratta, quindi, di chiedersi solo in che modo 
esse possano migliorare gli apprendimenti, oppure, se i media digitali possano far male o bene agli studenti, piuttosto, "occorre pensare alle tecnologie non nel senso di un'etica del rischio ma delle opportunità. [...] comprendere il valore e le potenzialità delle relazioni che a partire da esse e con esse si stabiliscono" (Rivoltella \& Rossi, 2019, pp. 6-7); occorre altresì verificare, preventivamente, quali e quante tecnologie possono realmente definirsi "inclusive" amplificando le risorse fisiche, sensoriali e cognitive della persona sostenendola nel suo diritto di scegliere e decidere in ogni suo contesto di vita, perchè migliorare la qualità della vita delle persone e, di conseguenza, predisporre per esse reali occasioni di inclusione, può rendere tutti capaci di operare delle scelte e di realizzare un progetto individuale, consapevoli che questa "spinta all'agire autodeterminato caratterizza tutti gli individui, indipendentemente dalle loro abilità e competenze, ed è una delle condizioni di base su cui si fonda la qualità stessa della vita di ognuno" (Cottini, 2016, p. 16).

\section{Tecnologie assistive e Unione Europea}

Oggigiorno, nell'Unione Europea, le tecnologie assistive (Besio, 2006) sviluppate per studenti con disabilità (Besio, 2019) sono progettate tenendo conto degli aspetti normativi, sanitari e demografici (EPRS, 2018a), in linea con la Classificazione Internazionale di Funzionamento, Disabilità e Salute (Pavone, 2015) e seguono le tendenze del mercato delle TIC per essere sicuri che tutte le nuove tecnologie possano fornire un supporto o un sollievo, in qualche modo, alle persone con disabilità (EPRS, 2018b). Promuovere un approccio pedagogico inclusivo è significativo per sottolineare le prospettive, le potenzialità e le opportunità delle tecnologie assistive (EPRS, 2018c), capire in che modo aumentano i vantaggi legali e socio-etici (EPRS, 2018d) e come contribuiscono a raggiungere l'assistive inclusion (EPRS, 2018e) per realizzare la piena inclusione (Pavone, 2015) nei paesi europei e in ogni nazione al mondo. Andando più nel dettaglio, gli studenti disabili possono portare le tecnologie assistive a scuola, i dislessici avere con sè un software di sintesi vocale (installato nel proprio smartphone, pc o tablet) o i discalculici avere in tasca la propria calcolatrice (che ora è un software integrato in ogni smartphone) per supportare i propri processi di insegnamento-apprendimento (Di Tore, 2016). In effetti, Il trattato su cui si fonda il funzionamento dell'Unione Europea affronta il tema cardine e fondativo, ovvero che costituisce il fondamento e che è alla base della stessa Unione Europea, della "non discriminazione" (EPRS, 2018a, p. 7) che se negli anni Cinquanta si riferiva principalmente all'elemento distintivo della nazionalità ora si è esteso a ogni elemento che contraddistingue l'individuo nella sua unicità. L'Unione Europea si impegna, pertanto, a tener in forte considerazione la prospettiva della disabilità in tutte le politiche $\mathrm{e}$ attività (ibidem). Oltre a queste legislazioni generali, esiste anche una legislazione sui diritti delle persone con disabilità in settori specifici, ciò include: le reti di trasporto, le telecomunicazioni e il mercato del lavoro (ibidem). Altre aree, invece, sono gestite direttamente dai singoli stati membri, ad esempio i sistemi di sicurezza sociale e i sistemi scolastici nazionali (ibidem). Ma volendo ancora soffermarsi, in modo generale, sul concetto di assistive technologies e di come esse possono alleviare la vita e le attività di studio e lavoro di tante persone diverse sia per età che per estrazione sociale e culturale, la Scientific Foresight Unit, dell'European Parliamentary Research Service, evidenzia che si possono identificare tre linee di tendenza per le assistive technologies (EPRS, 2018b, p. 46): 1) realizzare l'integrazione nella tecnologia mainstream; 2) favorire l'autonomia; 3) realizzare protesi biomediche. L'European Parliamentary Research Service propone quattro scenari entro i quali le assistive technologies possono svilupparsi (EPRS, 2018c, p. 
94) e tali scenari rifletteranno il livello di inclusione che la società avrà raggiunto e, pertanto, potranno essere diversi in ogni stato membro della comunità europea e forse, sarebbe lecito dire, diversi nelle stesse regioni e province degli stati membri. Questo produrrebbe una falsa inclusione, a macchia di leopardo, con gravi conseguenze in termini di potenzialità, trasporto e opportunità per tutti e ciascuno producendo fenomeni di migrazione che nascono per necessità di vivere in luoghi inclusivi che offrono maggiori opportunità ma producono la divisione di nuclei familiari e allontanano le persone che aspirano a una società più inclusiva dai propri affetti; pertanto la comunità europea promuove un procedere omogeneo e non a macchia di leopardo, quantomeno in Europa, di politiche inclusive che divengono realmente pratiche inclusive.

\section{Tecnologie e corporeità nella didattica}

L'attuale approccio nel settore delle scienze cognitive dell'Embodied Cognition (Johnson, 2017; Lakoff \& Johnson, 1999), a partire dai costrutti psico-pedagogici che ritenevano il fare del bambino e l'intelligenza senso-motrice (Dewey, 1933; 2014; Piaget, 1967) quale base per la costruzione degli apprendimenti, incentivati dal desiderio di scoperta e di partecipazione (Montessori, 1999; 1946/2000), colloca la corporeità al centro dell'agire educativo-didattico e riconosce in essa il substrato per lo sviluppo dei processi cognitivi. L'ipotesi di una relazione tra pedagogia e biologia, già suggerita dagli studi sulla bioeducazione (Frauenfelder \& Santoianni, 2002; Frauenfelder, Santoianni \& Striano, 2004) che individuano nel corpo un'innata disposizione all'apprendimento, ha offerto suggestive riflessioni sul rapporto esistente tra esperienza individuale e dimensione ambientale, spostando il focus delle indagini sul ruolo adattivo dell'individuo e sulla funzione educativa del contesto "perché è proprio nei contesti, negli ambienti e nelle relazioni dell'educazione, della formazione, dell'istruzione che la mente attiva il proprio potenziale apprenditivo attraverso specifiche reti neurali" (Frauenfelder et al., 2004, p. 61). Questi studi, declinati nell'ambito delle scienze dell'educazione e della formazione, hanno reso possibile un' analisi multidimensionale delle esperienze educative vissute dal soggetto che apprende attribuendo significatività all'intersoggettività della mente e del corpo situati nei contesti (Gallese \& Ammaniti, 2014), oltre a riconoscere l'esistenza di un'interazione costante tra sistemi emotivi e cognitivi (Damasio, 1995; 1999) e il ruolo della mediazione linguistica nel processo adattivo (Orefice, 2001, 2003). L'efficacia della centralità del corpo nell'ambito dei processi d'insegnamento-apprendimento risulta, difatti, strettamente connessa alla motivazione e al vissuto emotivo di colui che apprende: per Dehaene (2019) "un organismo passivo non impara, o impara molto poco. Imparare efficacemente significa rifiutare la passività, essere coinvolti, esplorare, generare attivamente delle ipotesi" (p. 218). Il dispositivo embodiment, dunque, può facilitare la costruzione della conoscenza grazie alla partecipazione attiva dell'alunno nelle attività didattiche, offrendogli la possibilità di vivere emozioni intense e profonde tali da agire come input di rinforzo e da determinare un coinvolgimento complessivo della sua persona nell'esperienza educativa (Gomez Paloma, 2004; 2009; 2013). Appare evidente come, i soli vincoli biologici non bastino a definire la specificità dell'essere umano piuttosto, nell'incessante interazione con l'ambiente, attraverso cui il corpo e il movimento lasciano tracce indelebili nelle strutture cerebrali di ciascun individuo, risultano essere determinanti l'insieme di esperienze vissute dal soggetto (Bruner, 2003; 2009). L'apprendimento umano presuppone una specifica natura sociale e si configura come un processo in cui la costruzione delle abilità mentali avviene anche in virtù dell'intensificarsi della comunicazione il cui veicolo di trasmissione, come affermava Vygotskij (1934/1992), non è rappresentato solo dal linguaggio ma anche 
dai suoi prodotti (istruzione, scienza, letteratura, tecnologia) che, parimenti, rendono possibile e orientano l'azione del bambino i cui problemi, anche i più pratici, possono essere risolti proprio mediante quest'unità di percezione, azione e linguaggio favorita tanto dagli strumenti della cultura quanto dagli aiuti che egli può ricevere dagli adulti nel delicato percorso dell'apprendere. Secondo Oliverio (2017), infatti, la mente, nel suo essere estesa ha il merito non solo di esteriorizzare "tramite il linguaggio complessi concetti che a loro volta strutturano il pensiero, ma sfrutta anche le più diverse tecnologie, da quelle più essenziali come una penna o un quaderno, a quelle più complesse come uno smartphone $\mathrm{o}$ un computer. Questi strumenti amplificano il potenziale della mente e agiscono a loro volta su di essa, plasmandola, dandole nuove forme e capacità" (pp. 102-103), soprattutto in quei casi in cui il linguaggio non è sufficiente e la persona necessita di un' "estensione" che le consenta di superare i propri confini e di relazionarsi con il mondo. Non è un caso, infatti, che gli uomini primitivi si siano serviti di segni e simboli per comunicare e che le loro prime forme di scrittura abbiano rappresentato, come avviene oggi per una penna, un foglio di carta o una tecnologia (Diamond, 1998), un modo per "scaricare memorie dal nostro cervello e affidarle ai media più disparati" (Oliverio, 2017, p. 104). Nell'ambito del filone di ricerca dell'embodiment education, gli studi volti ad indagare il rapporto tra tecnologia e corporeità, si concentrano anche sul modo in cui i dispositivi tecnologici possano "attivare processi senso-motori, spesso simulati, per gestire concetti, attività ed esperienze" (Rivoltella \& Rossi, 2019, p. 29) e, nel contempo, "replicano, potenziandole, le funzioni dei nostri sensi e dei nostri arti" lasciando al soggetto la possibilità di osservare, agire, dialogare e manipolarli con il proprio corpo (ivi, p. 72). In quanto parti integranti della didattica, le tecnologie possono determinare importanti ricadute in termini di apprendimento, socializzazione e inclusione per gli alunni che, in presenza di bisogni educativi speciali, possono necessitare, più degli altri, di un supporto capace di facilitare sia l'accesso ai contenuti disciplinari sia le relazioni. In entrambi i casi, il loro uso risulta imprescindibile dalla valorizzazione di tutti gli aspetti connessi all'embodiment: l'intelligenza del corpo quale dimensione trasversale attraverso cui apprendere nel rispetto degli stili cognitivi dello studente e del suo potenziale creativo (Gardner 1987; De Bono, 2001); la dimensione vicariante, ovvero, la possibilità per l'alunno di supplire ad alcuni deficit sensoriali, quando una facoltà sia perduta o compromessa. In questa direzione, la "vicarianza - lungi dall'essere un inconveniente - genererebbe, grazie al suo potenziale combinatorio, la diversità tra gli individui. Costituirebbe, inoltre, una riserva di capacità di adattamento" (Berthoz, 2015, p. 23) in un ambiente che "smette di essere concepito come un elemento neutro, una risorsa di stimoli freddamente rispecchiati nelle nostre rappresentazioni mentali, ma diventa piuttosto un carico di valori, di significati e di rimandi all'azione e all'interazione" (Caruana \& Borghi, 2016, p. 23); la funzione imitativa e simulativa di un gesto o di un'azione mediata dallo strumento ma resa possibile dai neuroni mirror (Rivoltella, 2014; Rizzolatti \& Sinigallia, 2007), grazie ai quali le due dimensioni neurali del "vedere" e dell" "agire" risultano perfettamente integrate, facilita la percezione del mondo esterno, attiva specifici circuiti neuronali e può favorire l'apprendimento di routine. La possibilità di utilizzare il corpo, il gesto ed il movimento nella loro accezione vicariante, compensativa e simulativa, mediata anche dalle tecnologie, non appartiene solo a colui che apprende, ma anche a colui che insegna: nel processo di insegnamentoapprendimento entrano in gioco, quindi, "corporeità didattiche" (Sibilio, 2011) che contribuiscono a delineare un sistema di relazioni e percorsi educativi e formativi che in maniera ricorsiva e alternativa si intersecano secondo traiettorie non lineari (Sibilio, 2014). 


\section{CAA e tecnologie assistive nella didattica inclusiva}

Accogliere il principio di educabilità dell'individuo, in una prospettiva neuroscientifica, significa non solo riconoscere nella corporeità e nel movimento le dimensioni attraverso cui incentivare lo sviluppo dei costrutti emotivi, relazionali e cognitivi dei propri allievi (Cottini, 2003; Moliteni, 2013) ma anche valorizzare quegli aspetti della didattica che possono incidere sul potenziale di plasticità di ciascuno (Edelman, 1995; Frauenfelder, 1986) e rendere le pratiche "più" o "meno" inclusive: si pensi alla preventiva organizzazione degli spazi, alla scelta di strumenti, strategie e metodologie, alla condivisione di tecniche di comunicazione, anche non verbali, che utilizzano un preciso sistema di segni (la LIS, ad esempio) o un insieme di sistemi simbolici basati su pittogrammi. In entrambi i casi il fulcro dell'azione e della relazione didattica è rappresentato da un corpo agito che attinge alle proprie risorse per esprimere emozioni, pensieri, bisogni e creare un ponte comunicativo con gli altri, oltre ad un canale "alternativo" nella costruzione dei propri saperi. Gli studi sulla comunicazione umana hanno dimostrato come, a prescindere dall'uso delle parole, sia possibile condividere con i nostri interlocutori una vasta gamma di contenuti (Bonaiuto \& Maricchiolo, 2009; Ricci Bitti \& Cortesi, 1977) e hanno individuato nel linguaggio del corpo (Argyle, 1992; Kendon, 1981), e nelle sequenze di segnali che lo contraddistinguono, una manifestazione dello stato emotivo vissuto dalla persona e un comune denominatore biologico tra le culture (Ekman, 2008; Friesen \& Ekman, 2007): espressioni del volto, gesti, movimenti del corpo, tono e ritmo della voce, modulazione della distanza interpersonale e contatto reciproco (Hall, 1969) evidenziano l' impossibilità di non comunicare laddove anche il silenzio risulta pregno di significati (Watzlawick, Beavin \& Jackson, 1971). Queste opportunità comunicative offerte dalla corporeità e dal movimento, costituiscono uno degli elementi costitutivi degli interventi di Comunicazione Aumentativa Alternativa (CAA) sia nei contesti clinici/riabilitativi sia in ambito educativo. Il modello della partecipazione sociale (Beukelman \& Mirenda, 2014; Sigafoos \& Mirenda, 2002), alla base di ogni progetto personalizzato di CAA, afferma l'importanza di un intervento precoce e propone di ampliare le occasioni comunicative in tutti i contesti di vita a partire dall'identificazione degli interessi e delle aree motivazionali della persona, dalla comprensione dei suoi segnali corporei e dall'introduzione di un ventaglio di scelte. Nello specifico, quest'area di ricerca e di pratica clinica, sin dalle sue origini, ha sposato l'idea che è possibile compensare la disabilità temporanea o permanente di persone con bisogni comunicativi complessi accogliendo e valorizzando i segnali del corpo (vocalizzi, movimenti oculari, gesti referenziali, ecc.) e sfruttando, nello stesso tempo, le opportunità inclusive e comunicative offerte da strumenti cartacei e tecnologici. Dei validi supporti alla didattica sono rappresentati dai Vocal Output Communication Aids (VOCAs), comunicatori con uscita vocale grazie ai quali è possibile registrare e, successivamente, riprodurre uno o più messaggi mediante un pulsante sul quale può anche essere applicato un simbolo o un' immagine. Grazie all'ancoraggio a supporti esterni e all'abbinamento ad interfacce o a giocattoli, l'alunno, oltre a comprendere il legame di causa-effetto esistente tra la pressione dell'ausilio e l'attivazione del gioco o l'emissione del messaggio, è sostenuto nella comunicazione che può divenire sempre più articolata (si pensi a comunicatori che consentono la registrazione di 20 messaggi da riascoltare in sequenza) e nell'interazione con l'adulto e i compagni. Nell'ambito dei software disponibili, ad esempio, uno dei programmi grafici più noti per la produzione dei materiali di comunicazione è costituito dal Boardmaker, tra l'altro utilizzato nell'esperienza che si descriverà in seguito in quanto già disponibile nell'istituzione scolastica. Con oltre 6500 simboli e oltre 250 griglie predisposte per il loro inserimento, il software consente la costruzione dei principali 
materiali per la CAA (tabelle di comunicazione, strisce, agende visive, traduzione di testi), grazie anche alla flessibilità delle sue impostazioni che rendono possibili modifiche al colore e alle dimensione dei simboli, la scelta dei modelli per l'inserimento dei pittogrammi, la stampa e la plastificazione. L'ampia libreria contenuta al suo interno, inoltre, facilita la personalizzazione della didattica rendendo agevole l'introduzione di foto, disegni e immagini da archiviare in categorie accessibili in qualsiasi momento. Il sistema simbolico di base è rappresentato dal Picture Communication Symbols (PCS) che si caratterizza per una chiara raffigurazione del significato (immagine con parola scritta in minuscolo). Date le esigenze degli utenti e degli operatori del settore, risulta essere in continua espansione: periodicamente vengono raccolti nuovi pittogrammi e ogni due anni circa è possibile accedere ad un nuovo sistema di simboli aggiornato. Tuttavia, il PCS non consente l'introduzione nel testo di articoli, preposizioni o di simboli che possano esprimere il genere e il numero dei sostantivi e dei tempi verbali, contribuendo così alla traduzione di elaborati anche complessi che è invece possibile con il Widgit Literacy Symbols (WLS), conosciuto anche con il nome Widgit. Questo set di pittogrammi, tra i più noti ed usati dagli utenti e dagli operatori della CAA per la sua grafica chiara, essenziale e adatta anche a persone adulte, è contenuto nel software Symwriter. I sistemi simbolici e i software citati, purtroppo, prevedono delle limitazioni nel loro uso e nella distribuzione pubblica, limitando fortemente la condivisione dei materiali prodotti in ambito scolastico. Per queste ragioni, in tutte le scuole in cui non è disponibile uno dei due software, è possibile ovviare al problema del copyright attingendo ai simboli e ai materiali open source disponibili sul sito ARASAAC (https://arasaac.org/). Queste tecnologie, utilizzate in un progetto personalizzato di CAA in ambito educativo, possono avere un ruolo importante nel sostenere l'autonomia degli alunni con bisogni educativi speciali e rappresentare strumenti capaci di rispondere alle loro diverse esigenze funzionali; quando inserite all'interno della programmazione didattica debbono essere opportunamente scelte ed integrate alle metodologie più efficaci con lo scopo di perseguire il successo formativo di tutti e di ciascuno (Besio, 2005).

\section{L'uso di GRID3 e del comunicatore oculare}

Nella prospettiva di Ferri (2018), la ridefinizione di un nuovo sistema scolastico dovrebbe sempre implicare una riprogettazione tale da creare, ovunque, delle scuole e una didattica aumentata dalle tecnologie (Ferri, 2013) laddove, le componenti tecnologiche (software e device hardware) si integrino a metodologie e strategie didattiche in originali setting d'aula nei quali, quest' uso combinato di dispositivi tecnologici che si distinguono per le proprie opzioni visive, uditive e di manipolazione possa arricchire la didattica e offrire opportunità inclusive per ciascun alunno. Nell'esperienza didattica a distanza, che ha interessato la classe terza di una scuola primaria romana, l'intervento educativo in CAA predisposto per un'alunna con un ritardo cognitivo grave, ipotonia e assenza di linguaggio verbale, ha richiesto una rivisitazione complessiva della sua originaria impostazione in mancanza di spazi di condivisione e in assenza di prompt fisici dei principali partner comunicativi (docenti curricolari e di sostegno, operatore OEPA, assistente alla comunicazione). Secondo quanto disposto dalla N.M. n. 1041/2020, che ha richiesto una rimodulazione dei Piani Educativi Individualizzati (PEI), il Gruppo di Lavoro Operativo per l'inclusione scolastica (GLO), riunitosi per fronteggiare l'inaspettata emergenza educativa, nel predisporre l'insieme degli interventi da attuarsi a distanza attraverso la piattaforma Teams) e il registro elettronico Axsios, ha concordato di costruire l'intero percorso didattico a partire da un potenziamento delle abilità corporeo-cinestesiche dell'alunna (in particolare 
la velocità di sguardo) attraverso l'uso di un comunicatore oculare con uscita in voce, il cui training era iniziato già nei mesi precedenti. Nello specifico, la tecnologia in oggetto è composta da: comunicatore oculare dinamico - tablet 19" con accesso mediante emulatore di mouse con lo sguardo, sistema di ancoraggio da tavolo per il posizionamento, sintesi vocale e software di comunicazione GRID3 (Figura 1). La sintesi vocale disponibile è sapi5 che non si basa sulle classiche voci sintetiche proposte da Microsoft quali Zira, Elsa o simili, ma si differenzia per essere la prima sintesi con voce da bambina (https://www.helpicare.com/prodotto/aurora/) compatibile con tutti i software per la comunicazione. Molto più accattivante di quelle disponibili sul $\mathrm{pc}$ o che è possibile scaricare gratuitamente, questa tipologia di sintesi, presente nel comunicatore oculare, consente all'alunno una maggiore autonomia nella lettura dei testi in CAA, una migliore comprensione dei contenuti e facilita le relazioni con gli altri grazie all'opportunità di integrare all'ascolto dei testi il supporto di immagini, foto e simboli. Anche in questo caso, come per molti software e sistemi simbolici, è probabile che in futuro ci siano delle alternative open source per andare incontro alla necessità di spending review che la scuola impone.

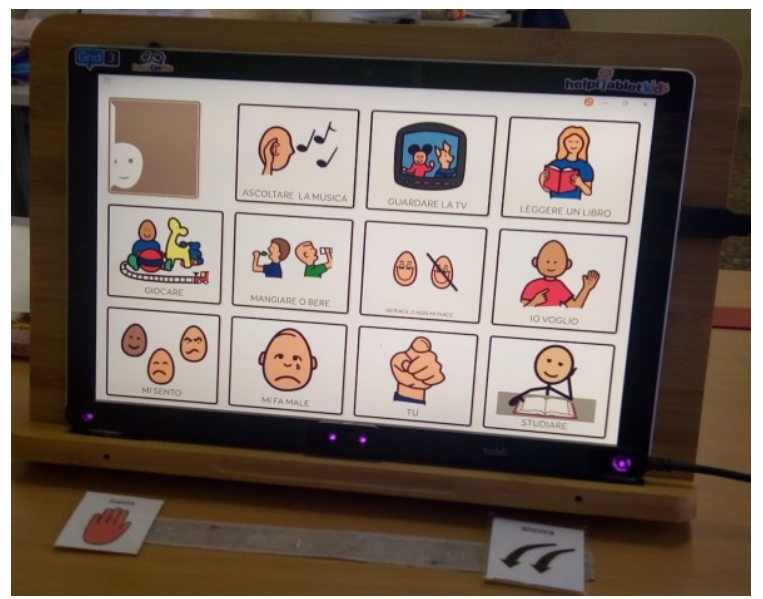

Figura 1. Schermata Home del comunicatore oculare dinamico con Software GRID3.

Il software di comunicazione GRID3 (Figura 1), inoltre, è costituito da sistemi funzionali alla comunicazione sia alfabetica che simbolica, da un'ampia libreria di pittogrammi Widgit per la realizzazione di sistemi dinamici di comunicazione e di tabelle. Rispetto alle precedenti versioni, l'introduzione del soggetto "io" nella costruzione della frase ha reso possibile la coniugazione automatica dei verbi consentendo agli utenti, che non possono accedere al codice alfabetico, l'opportunità di comunicare con gli altri attraverso frasi corrette sul piano grammaticale sia nell'interazione face to face, sia nelle relazioni a distanza. In questo caso, la predisposizione di un accesso facilitato alla mail, ai principali social e sistemi di messaggistica istantanea facilita le relazioni e rende fattibile la scrittura dei messaggi, la lettura e l'ascolto grazie alla pressione di pochissimi tasti. In ambito scolastico, questa tecnologia offre alla persona l'opportunità di esprimere richieste, condividere emozioni e interessi, effettuare delle scelte grazie alla presenza di macro-aree rappresentate da celle molto grandi, visibili sul monitor del tablet, e accessibili mediante puntamento oculare, touch o sensori esterni. La semplicità di utilizzo del software rende possibile la modifica delle griglie, l'aggiornamento dei contenuti in CAA grazie ai diversi livelli di apprendimenti e all'interfaccia touch-friendly; quest'ultima consente l'accesso ai contenuti con una leggera pressione della mano, delle dita o mediante lo sguardo: in questo caso, è necessario trovare la giusta distanza tra il corpo dell'utente e l'ausilio. L'avvenuto 
riconoscimento oculare sarà indicato da un volto bianco, visibile nella cella in alto a sinistra; al contrario, l'impossibilità di fissare lo schermo a causa di continui movimenti del capo dell'alunno, oppure un'errata calibrazione della periferica di tracciamento oculare, saranno segnalate dalla presenza di un viso rosso. Sebbene il suo utilizzo sia semplice ed intuitivo sia per l'utente con BCC sia per il partner comunicativo (familiare, educatore, insegnante, terapista) l'inserimento nella didattica a distanza ha richiesto delle preventive modifiche relativamente all'organizzazione degli spazi, alla predisposizione degli strumenti cartacei in CAA, agli obiettivi educativo-didattici da perseguire, alla scelta delle strategie e delle attività supportate dalle tecnologie.

\subsection{Organizzazione dell'intervento didattico in CAA a distanza}

In continuità con il percorso educativo e didattico in presenza che ha previsto, sin dal primo anno, un'organizzazione degli spazi scolastici con lo scopo di renderli accessibili sul piano comunicativo, mediante la distribuzione di PCS in tutti gli ambienti come l'aula, la mensa, la palestra e il giardino, lo stesso contesto familiare ha subito una nuova rimodulazione nel periodo del lockdown, includendo al suo interno: un banco incavo con strisce di velcro, utili alla predisposizione dei pittogrammi; la distribuzione di simboli in ogni ambiente domestico; l'etichettamento di tutti i materiali e degli oggetti funzionali allo svolgimento delle attività scolastiche in modalità sincrona; l'accessibilità del comunicatore e degli strumenti di comunicazione. Lo svolgimento delle attività didattiche sempre nello stesso luogo e la difficoltà dell'alunna di discriminare le varie fasi della giornata, in precedenza scandite da routine giornaliere condivise dal gruppo dei pari (ad esempio l'entrata in classe, la lettura del calendario in CAA, l'appello e la ricreazione) ha richiesto la predisposizione, anche nel contesto domestico, di ulteriori aiuti visivi che, rispetto alla variabilità della comunicazione verbale, costituiscono per la persona un elemento stabile e invariabile sul piano percettivo. Le agende e le strisce visive (Figura 2) hanno permesso di: offrire un supporto visivo costante ma, nello stesso tempo, modificabile a seconda delle attività; migliorare l'attenzione sostenuta fortemente inficiata dalla presenza dello schermo; integrare la comunicazione veicolata anche da tabelle a tema utili nello svolgimento delle attività didattiche.

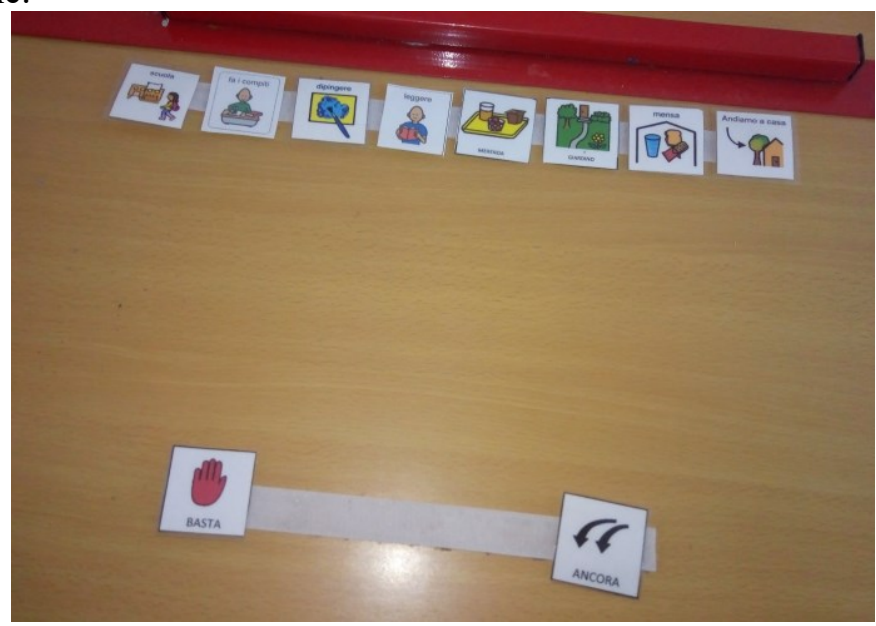

Figura 2. Esempio di striscia visiva.

L'uso del comunicatore oculare con software GRID3, parte integrante delle attività è stato finalizzato al perseguimento dei seguenti obiettivi educativo-didattici: comunicare bisogni, 
desideri, emozioni ed interessi mediante la sintesi vocale; facilitare l'immediatezza e la velocità nell'interazione con il gruppo classe a distanza, migliorandone la partecipazione; favorire l'autodeterminazione e la possibilità di scelta di un'attività; migliorare l'acquisizione dei contenuti afferenti alle diverse aree disciplinari, accessibili mediante le griglie preventivamente predisposte; ampliare il vocabolario di simboli in CAA. I contenuti curricolari, i giochi e i testi delle canzoni, preparati molti giorni prima della lezione calendarizzata, sono stati tradotti in CAA e inseriti in presentazioni in power point, con lo scopo di consentire all'alunna la consultazione e la memorizzazione in modalità asincrona (dal tablet e dal pc) e la condivisione con i compagni in modalità sincrona: in quest'ultimo caso, la condivisione dello schermo, la sintesi con voce da bambina e la mediazione dell'assistente alla comunicazione hanno reso più facile l'interazione con i compagni e $i$ docenti riducendo quel gap derivante dall'assenza di linguaggio verbale (Figura 3). Allo stesso modo, i libri illustrati con testo scritto in simboli (In-book) in formato pdf, leggibili sullo schermo, hanno rappresentato un ulteriore sostegno alla comunicazione e un' occasione di partecipazione.

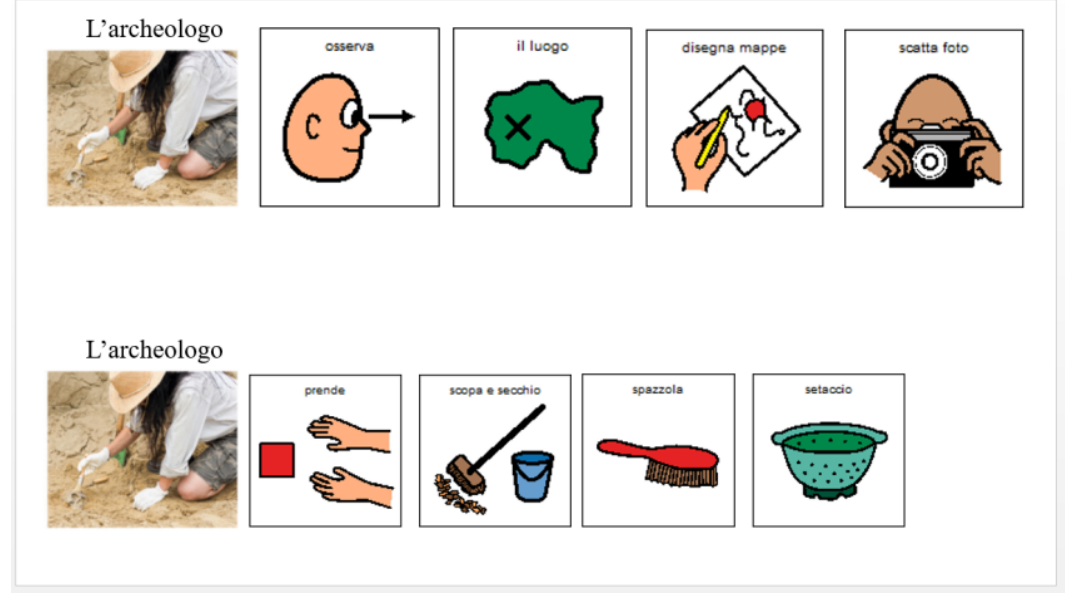

Figura 3. Esempio di una lezione di storia in ppt accesibile tramite PC e comunicatore oculare.

\section{Conclusioni}

Le tecnologie assistive possono consentire alla persona maggiori occasioni di partecipazione, autodeterminazione e sviluppo del proprio potenziale in ogni contesto di vita, ivi compreso quello educativo. L'impossibilità di utilizzare il linguaggio nella comunicazione con gli altri, le difficoltà motorie e un ritardo cognitivo rilevante, possono rappresentare motivi di esclusione scolastica se l'alunno non è opportunamente supportato da specifici sussidi e ausili che ne favoriscano le relazioni e gli apprendimenti. Nell'esperienza in oggetto, il comunicatore oculare e gli strumenti cartacei propri della CAA hanno offerto l'opportunità di lavorare sul miglioramento delle autonomie (personali, sociali e scolastiche) e sull'ampliamento delle capacità comunicative e relazionali. La traduzione dei contenuti disciplinari in CAA, disponibili su piattaforma, ha rappresentato anche per gli alunni della classe un supporto visivo nella memorizzazione delle lezioni in modalità asincrona, oltre ad un'occasione per ampliare il proprio vocabolario in simboli indispensabile nelle interazioni con la compagna. La didattica a distanza, inoltre, pur modificando le modalità di relazione con l'altro ha evidenziato quanto il connubio tra corporeità e tecnologia possa offrire opportunità comunicative e apprenditive anche in presenza di disabilità gravi, evidenziando come la scuola e le politiche inclusive possano 
determinare quale dei quattro scenari, ipotizzati dall'European Parliamentary Research Service (EPRS, 2018c, p. 94) possa realmente concretizzarsi in futuro, mostrando l'effettivo livello di inclusione raggiunto dalla società.

\section{Riferimenti bibliografici}

Argyle, M. (1992). Il corpo e il suo linguaggio. Studio sulla comunicazione non verbale (M. Montesano, Trans.). Bologna: Zanichelli (Original work published 1975).

Bertagna, G. (2020). Reinventare la scuola Un'agenda per cambiare il sistema di istruzione e formazione a partire dall'emergenza Covid-19. Roma: Studium.

Berthoz, A. (2011). La semplessità (F. Niola, Trans.). Torino: Codice (Original work published 2009).

Berthoz, A. (2015). La vicarianza. Il nostro cervello creatore di mondi (S. Ferraresi, Trans.). Torino: Codice (Original work published 2013).

Besio, S. (2005). Tecnologie assistive per la disabilità. Con CD-ROM: Risorse sulle tecnologie per la disabilità. Lecce: Pensa Multimedia.

Besio, S. (2019). Tecnologie Assistive. In L. D’Alonzo (eds.), Dizionario di Pedagogia Speciale (pp. 356-364). Brescia: Scholé.

Beukelman, D. R., \& Mirenda, P. (2014). Manuale di Comunicazione Aumentativa e Alternativa. Interventi per bambini ed adulti con complessi bisogni comunicativi (Verruggio, G. et al., Trans). Trento: Erickson (Original work published 2013).

Boardmaker software http://www.auxilia.it/site/lang/it-IT/page/18/product/164 (ver. 19.12.2020).

Bonaiuto, M., \& Maricchiolo, F. (2009). La comunicazione non verbale. Firenze: Carocci.

Bruner, J. (2003). La mente a più dimensioni (M. Capitella, Trans.). Roma-Bari: Laterza (Original work published 1986).

Bruner, J. (2009). Il pensiero. Strategie e categorie (E. Riverso, Trans.). Roma: Armando Editore (Original work published 2009).

Calvani, A. (2007). Tecnologia, scuola, processi cognitivi. Per una ecologia dell'apprendere. Milano: FrancoAngeli.

Caruana, F., \& Borghi, A. (2016). Il cervello in azione. Introduzione alle nuove scienze della mente. Bologna: il Mulino.

Cottini, L., (2003). Psicomotricità. Valutazione e metodi di intervento. Roma: Carocci.

Cottini, L. (2016). L'autodeterminazione nelle persone con disabilità: percorsi educativi per realizzarla. Trento: Erickson.

Cottini, L. (2017). Didattica speciale ed inclusione scolastica. Roma: Carocci.

Damasio, A. R. (1995). L'errore di Cartesio. Emozione, ragione e cervello umano (F. Macaluso, Trans.). Milano: Adelphi (Original work published 1994). 
Damasio, A.R. (1999). The Feeling of What Happens: body and emotion in the making of consciousness. New York: Harvet Edition.

De Bono, E. (2001). Creatività e pensiero laterale. Manuale di pratica della fantasia ( $\mathrm{F}$. Brunelli, Trans.). Milano: Biblioteca Univ. Rizzoli (Original work published 1970).

Dehaene, S. (2019). Imparare. Il talento del cervello, la sfida delle macchine (L. Ducceschi, Trans.). Milano: Raffello Cortina (Original work published 2019).

Dewey, J. (1933). How we think: A restatement of the relation of reflective thinking to the educative process. New York: D.C. Heath and Company.

Dewey, J. (2014). Esperienza ed educazione (E. Codignola, Trans.). Milano: Raffaello Cortina (Original work published 2008).

Diamond, J. (1998). Armi, acciaio e malattie (C. Civalleri, Trans.). Milano: Einaudi (Original work published 1997).

Di Tore, S. (2016). La tecnologia della parola, didattica inclusiva e lettura. Milano: Franco Angeli.

Dovigo, F. (2008). L'Index per l'inclusione: una proposta per lo sviluppo inclusivo della scuola. In: T. Booth \& M. Ainscow (eds.), L'Index per l'inclusione. Promuovere l'apprendimento e la partecipazione nella scuola (E. Valtellina, Trans.) (pp. 7-41). Trento: Erickson (Original work published 2002).

Edelman, G.M. (1995). Darwinismo neurale. La teoria della selezione dei gruppi neuronali (S. Ferraresi, Trans.). Torino: Einaudi (Original work published 1987).

Ekman, P. (2008). Te lo leggo in faccia. Riconoscere le emozioni anche quando sono nascoste (A. Basso, Trans.).Torino: Amrita (Original work published 2004).

EPRS. European Parliamentary Research Service, Scientific Foresight Unit (2018a). Assistive technologies for people with disabilities Part I: Regulatory, health and demographic.

aspects.

https://www.europarl.europa.eu/RegData/etudes/IDAN/2018/603218/EPRS IDA (2018)603218(ANN1) EN.pdf (ver. 12.12.2020).

EPRS. European Parliamentary Research Service, Scientific Foresight Unit (2018b). Assistive technologies for people with disabilities Part II: Current and emerging technologies.

https://www.europarl.europa.eu/RegData/etudes/IDAN/2018/603218/EPRS IDA (2018)603218(ANN2)_EN.pdf (ver. 12.12.2020).

EPRS. European Parliamentary Research Service, Scientific Foresight Unit (2018c). Assistive Part III: Perspectives, needs and opportunities. https://www.europarl.europa.eu/RegData/etudes/IDAN/2018/603218/EPRS IDA (2018)603218(ANN3) EN.pdf (ver. 12.12.2020).

EPRS. European Parliamentary Research Service, Scientific Foresight Unit (2018d). Assistive Part IV: Legal and socio-ethical perspectives. https://www.europarl.europa.eu/RegData/etudes/IDAN/2018/603218/EPRS_IDA (2018)603218(ANN4)_EN.pdf (ver. 12.12.2020).

EPRS. European Parliamentary Research Service, Scientific Foresight Unit (2018e). Assistive Inclusion, In-Depth Analysis. 
https://www.itas.kit.edu/downloads/projekt/projekt nier16 asstech_EPRS IDA $2018 \quad 603218$ EN.PDF (ver. 12.12.2020).

Ferri, P. (2013). La scuola 2.0. Verso una didattica aumentata dalle tecnologie. Parma: Spaggiari.

Ferri, P., \& Moriggi, S. (2018). A scuola con le tecnologie. Manuale di didattica digitalmente aumentata. Milano: Mondadori.

Frauenfelder, E. (1986). La prospettiva educativa tra biologia e cultura. Napoli: Liguori.

Frauenfelder, E., \& Santoianni, F. (2002). Le scienze bioeducative. Prospettive di ricerca. Napoli: Liguori.

Frauenfelder, E., Santoianni, F., \& Striano, M. (2004). Introduzione alle scienze bioeducative. Roma-Bari: Laterza.

Friesen, W.V., \& Ekman, P., (2007). Giù la maschera. Come riconoscere le emozioni dall'espressione del viso (G. Noferi,Trans.). Roma: Giunti (Original work published 2003).

Gallese, V., \& Ammaniti, M. (2014). La nascita dell'intersoggettività. Lo sviluppo del sé tra psicodinamica e neurobiologia. Milano: Raffaello Cortina.

Gardner, H. (1987). Formae mentis. Saggio sulla pluralità dell'intelligenza (L. Sosio, Trans.). Milano: Feltrinelli (Original work published 1983).

Gomez Paloma, F. (2004). Corporeità ed emozioni. Una didattica psicomotoria per la costruzione del saper... essere. Napoli: Guida.

Gomez Paloma, F. (2009). Corporeità, didattica e apprendimento. Le nuove neuroscienze dell'educazione. Salerno: Edisud.

Gomez Paloma, F. (2013). Embodied Cognitive Science: Atti incarnati della didattica. Roma: Nuova Cultura.

GRID3 software https://www.helpicare.com/prodotto/the-grid-3/ (ver. 19.12.2020).

Hall, E.T. (1969). Il linguaggio silenzioso (G. Celati, Trans.). Roma: Bompiani (Original work published 1967).

Ianes, D., \& Macchia, D. (2008). Didattica per i bisogni educativi speciali. Strategie e buone prassi di sostegno inclusivo. Trento: Erickson.

Ianes, D. (2015). Bisogni educativi speciali e inclusione: valutare le reali necessità e attivare tutte le risorse. Trento: Erickson.

Johnson, M. (2017). Embodied Mind, Meaning, and Reason. How Our Bodies Give Rise to Understanding. Chicago: The University of Chicago Press.

Kendon, A. (1981). Nonverbal Communication. Interaction and Gesture. Mouton: The Hague.

Lakoff, G., \& Johnson, M. (1999). Philosophyin the Flesh. The Embodied Mind \& its Challenge to Western Thought. New York: Basic Book.

Laurillard, D. (2014). Insegnamento come scienza della progettazione. Costruire modelli pedagogici per apprendere con le tecnologie (P.G. Rossi, Trans.). Milano: FrancoAngeli (Original work published 2012). 
Lazzari, M. (2017). Istituzioni di tecnologia didattica. Roma: Studium.

MIUR. Ministero dell'Istruzione, dell'Università e della Ricerca (2012). Direttiva Ministeriale del 27 Dicembre 2012. Strumenti d'intervento per alunni con bisogni educativi speciali e organizzazione territoriale per l'inclusione scolastica. https://www.miur.gov.it/documents/20182/0/Direttiva+Ministeriale+27+Dicembr e+2012.pdf/e1 ee3673-cf97-441c-b14d 7ae5f386c78c? version $=1.1 \& \mathrm{t}=1496144766837$ (ver. 27.12.2012).

MIUR. Ministero dell'Istruzione, dell'Università e della Ricerca (2013). Circolare ministeriale del 06 Marzo 2013, n. 8. Strumenti di intervento per alunni con bisogni educativi speciali (BES).http://bes.indire.it/?page_id=3898 (ver. 13.12.2020).

Moliterni, P. (2013). Didattica e scienze motorie. Roma: Armando.

Montessori, M. (1999). La mente del bambino. Milano: Garzanti.

Montessori, M. (2000). Educazione per un mondo nuovo (M. A. Magrini, Trans.). Milano: Garzanti (Original work published 1946).

Nota Ministero dell'Istruzione - Dipartimento per il sistema educativo di istruzione e di formazione - prot. 1041 del 15 giugno 2020 relativa a "Piani Educativi Individualizzati e inclusione". https://www.istruzioneer.gov.it/2020/06/15/pianieducativi-individualizzati-e-inclusione-nota-ministero-istruzione/) (ver. 19.12.2020).

Oliverio, A. (2017). Il cervello che impara. Neuropedagogia dall'infanzia alla vecchiaia. Firenze: Giunti.

Orefice, P. (2001). I domini conoscitivi. Origine, natura e sviluppo dei saperi dell'Homo sapiens sapiens. Roma: Carocci.

Orefice, P. (2003). La formazione di specie. Per la liberazione del potenziale di conoscenza del sentire e del pensare. Roma: Guerini Studio.

Pavone, E.M. (2015). Scuola e bisogni educativi speciali. Milano: Mondadori.

Piaget, J. (1967). Lo sviluppo mentale del bambino e altri studi di psicologia (E. Zamorani, Trans.). Torino: Einaudi (Original work published 1964).

Ricci Bitti, S., \& Cortesi, P.E. (1977). Comportamento non verbale e comunicazione. Bologna: il Mulino.

Rizzolatti, G., \& Sinigallia (2007). So quello che fai. Il cervello che agisce e i neuroni specchio. Milano: Raffaello Cortina.

Rivoltella, P.C. (2014). La previsione. Neuroscienze, apprendimento, didattica. Brescia: Morcelliana.

Rivoltella, P.C. (2017). La comunicazione e le relazioni didattiche. In P.C. Rivoltella \& P.G. Rossi (eds.), L'agire didattico. Manuale per l'insegnante (pp. 71-86). Brescia: La Scuola.

Rivoltella, P.C. (2017). Tecnologie di comunità. Brescia: La Scuola.

Rivoltella, P.C., \& Rossi, P.G. (2019). Il corpo e la macchina. Tecnologia, cultura, educazione. Brescia: Morcelliana. 
Sibilio, M. (2011). Corporeità didattiche: i significati del corpo e del movimento nella ricerca didattica. In M. Sibilio (ed.), Il corpo e il movimento nella ricerca didattica. Indirizzi scientifico-disciplinari e chiavi teorico-argomentative (pp. 47-69). Napoli: Liguori.

Sibilio, M. (2014). La didattica semplessa. Napoli: Liguori.

Sibilio, M. (2017a). Corpo e cognizione nella didattica. In P.C. Rivoltella \& P.G. Rossi (eds.), L'agire didattico. Manuale per l'insegnante (pp. 51-70). Brescia: La Scuola.

Sibilio, M. (2017b). Vicarianza e didattica. Corpo, cognizione, insegnamento. Brescia: La Scuola.

Sigafoos, J., \& Mirenda, P., (2002). Strengthening communication behaviours for gaining access to desired items and activities. In Reichle, J., Beukelman, D., \& Light, J., (Eds), Exemplary strategies for beginning communicators (pp. 123-156). Baltimore, MD: Paul Broo.

Symwriter Software, http://symwriter.auxilia.it/index.php/2016-02-02-12-57-59/isimboli-widgit (ver. 23.12.2020).

Vygotskij, L.S. (1999). Pensiero e linguaggio. Ricerche psicologiche (L. Mecacci, Trans.). Roma-Bari: Laterza (Original work published 1934).

Watzlawick, P., Beavin, J.H., \& Jackson, D.D. (1971). Pragmatica della comunicazione umana. Studio dei modelli interattivi delle patologie e dei paradossi (M. Ferretti, Trans.). Roma: Astrolabio-Ubaldini. (Original work published 1978).

Weick, K.E., \& Sutcliffe, K.M. (2010). Governare l'inatteso - Organizzazioni capaci di affrontare le crisi con successo (F. Dovigo, Trans.). Milano: Raffaello Cortina (Original work published 2009). 